05

\title{
Generation of Coherent Extreme Ultraviolet Radiation in an Air Gas Cell with a High Power Femtosecond Laser System*
}

\section{(C) Muhammed Sayrac}

Department of Nanotechnology Engineering, Sivas Cumhuriyet University,

Sivas, Turkey

e-mail: muhammedsayrac@cumhuriyet.edu.tr

Received August 04, 2020

Revised October 28, 2020

Accepted January 11, 2021

High harmonic generation (HHG) in air medium is experimentally produced by using a state-of-the-art high power laser system. The harmonic orders up to 35th harmonic are well observed. Optical power dependence of harmonic spectrum is obtained. Dynamics of a free electron in an intense laser field presents good agreement with the experimental results. The air medium is used as an interaction medium since air is a ready source in abundance. The harmonic spectral distribution is similar to that obtained in noble gases. Experimental results of harmonics produced in air medium follows same trends in terms of plateau region and cutoff harmonic order, which extends and enhances with increasing laser field.

Keywords: Extreme ultraviolet radiation, high harmonic generation, femtosecond laser, air, gas cell, three-step model, ionization.

* Полный текст статьи опубликован в „Optics and Spectroscopy“ 2021 V. 129. N 6. 